\title{
THE LATTICE OF PATTERNS INDUCED BY A POSITIVE CONE OF FUNCTIONS
}

\author{
BY
}

\author{
D. J. HARTFIEL AND C. J. MAXSON
}

\begin{abstract}
This paper contains a study of a special type of lattice which arises by considering the supports of functions in a positive cone of functions. It is shown that many known combinatorial structures provide examples of such a lattice. The basic problem addressed in the paper is that of determining the structure of this lattice.
\end{abstract}

Introduction. This work contains a study of a partially ordered set induced by a positive cone of functions. For this, let $n$ be a positive integer and $\mathcal{C}=\left\{x_{1}, x_{2}, \ldots, x_{n}\right\}$ a set of $n$ distinct points. Consider $[\mathcal{C}, R]$ the set of all

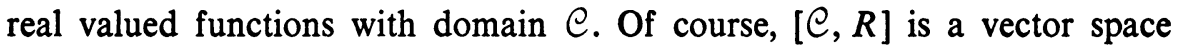
under the usual operations of addition and scalar multiplication.

Let $V$ be a subspace of $[\mathcal{C}, R]$ with $\delta=\{f \in V \mid f(x) \geqslant 0$ for all $x \in \mathcal{C}\}$. Any nonzero $f$ in $\mathcal{S}$ is called an assignment. These assignments can be used to induce patterns on $C$ by considering their supports, $|f|=\{x \mid f(x) \neq 0\}$. The set $\mathscr{D}(\mathcal{S})$ of all such patterns together with $\varnothing$ is then a partially ordered set under the inclusion relation.

As will be shown in the text, well-known matrix patterns that arise in this setting are those of irreducibility and full indecomposability. Relative to graph theory, we will show that strongly connected digraphs also arise in this manner. Several other patterns, perhaps not as well known as these, will also be given.

The partially ordered set $\mathscr{D}(\mathcal{S})$ is thus of some importance in each of these areas. It is our intent to give a study of these patterns. In particular, we will show that $\mathscr{D}(\mathcal{S})$ is a special type of lattice which we call an ideal complemented lattice. Further, some of the basic structure of this lattice will be given.

1. The ideal complemented lattice $\mathscr{D}(\delta)$. The purpose of this section is to show that the partially ordered set of patterns $\mathscr{D}(\delta)$ is in fact a special type of lattice. This will be accomplished by noting that since $|f+h|=|f| \cup|h|$ for all $f, h \in \mathcal{S}$ it follows that $\mathscr{D}(\mathcal{S})$ is a join semilattice and $|\cdot|: \mathcal{S} \rightarrow \mathscr{D}(\mathcal{S})$

Received by the editors March 18, 1975.

AMS (MOS) subject classifications (1970). Primary 05B20; Secondary 05B30, 06A25.

Key words and phrases. Combinatorial structure, lattice, positive cone.

- American Mathematical Society 1977 
a join homomorphism. This join homomorphism then provides the link between $\mathcal{S}$ and $\mathscr{D}(\mathcal{S})$ which allows the investigation of $\mathscr{D}(\mathcal{S})$ by algebraic techniques applied to $\delta$.

The following major tool in this investigation is modelled on the Birkhoffvon Neumann Theorem on doubly stochastic matrices.

LEMMA 1.1. For any assignment $f \in \mathcal{S}$, and $h \in V$ with $\varnothing \neq|h| \subseteq|f|$ there is a nonzero number $\lambda$ such that $f-\lambda h \in \mathcal{S}$ with $|f-\lambda h| \subsetneq|f|$.

Proof. Let $|h|=\left\{x_{1}, \ldots, x_{t}\right\}$. Then there exist nonzero reals $\lambda_{k}, k=1$, $2, \ldots, t$, such that $h\left(x_{k}\right)=\lambda_{k} f\left(x_{k}\right)$. Without loss of generality we suppose that $\max _{k} \lambda_{k}=\lambda_{k_{0}}>0$ so that $h\left(x_{k}\right)=\lambda_{k} f\left(x_{k}\right) \leqslant \lambda_{k_{0}} f\left(x_{k}\right)$ with equality for $k=k_{0}$. Thus setting $\lambda=1 / \lambda_{k_{0}}, f-\lambda h \in \mathcal{S}$ and $(f-\lambda h)\left(x_{k_{0}}\right)=0$. It follows then that $|f-\lambda h| \subsetneq|f|$.

We call an assignment $f \in \mathcal{S}$ atomic if and only if $|f|$ is an atom in $\mathscr{D}(\mathcal{S})$. Our goal is to show that $\delta$ is completely determined by atomic assignments and thus, through the join homomorphism, conclude that $\mathscr{D}(\mathcal{\delta})$ is completely determined by its atoms. These atomic assignments can easily be characterized by their supports.

LEMMA 1.2. If $f$ and $h$ are atomic assignments in $\delta$ with $|f|=|h|$ then $f=\lambda h$ for some positive number $\lambda$.

Proof. As $|h| \subseteq|f|$, and $h, f \in \delta$, by the above lemma there is a positive number $\lambda$ such that $|f-\lambda h| \subsetneq|f|, f-\lambda h \in \mathcal{S}$. Since $|f|$ is an atom, $|f-\lambda h|$ $=\varnothing$, i.e., $f=\lambda h$.

Showing then that $\delta$ is completely determined by these atomic assignments, we add the following.

THEOREM 1.1. If $f$ is an assignment in $\delta$, then there are atomic assignments $f_{1}, \ldots, f_{t}$ such that $f=f_{1}+\cdots+f_{t}$.

PRoof. The proof is by induction on the cardinality of $|f|$, denoted card $|f|$. If card $|f|$ is least for all $f \in \mathcal{S}$ then $f$ is atomic and the theorem holds. Suppose then that the result holds for all assignments $h \in \mathcal{S}$ where $0<$ $\operatorname{card}|h|<s$ and let $f \in \mathcal{\delta}$ with $\operatorname{card}|f|=s$. If $f$ is atomic then the theorem holds. Thus suppose $f$ is not atomic.

In this case there is an atom $\left|\bar{f}_{1}\right|$ so that $\left|\bar{f}_{1}\right| \subsetneq|f|$. Thus, by Lemma 1.1, there is a positive number $\lambda_{1}$ such that $f-\lambda_{1} \bar{f}_{1}^{\neq} \in \mathcal{S}$ and $\left|f-\lambda_{1} \bar{f}_{1}\right| \subsetneq|f|$. Since card $\left|f-\lambda_{1} \bar{f}_{1}\right|<s$, by the induction hypothesis there are atomic assignments $f_{2}, f_{3}, \ldots, f_{t}$ such that $f-\lambda_{1} \bar{f}_{1}=f_{2}+\cdots+f_{t}$. Setting $\lambda_{1} \bar{f}_{1}=$ $f_{1}$ the result follows.

In fact, if $h$ and $f$ are assignments in $\mathcal{S}$ with $|h| \subseteq|f|, h$ can be built up to $f$ by the addition of atomic assignments. 
COROLLARY 1.1. If $h$ and $f$ are assignments in $\delta$, with $|h| \subseteq|f|$, then there exist atomic assignments $f_{1}, \ldots, f_{t}$ and a positive number $\lambda$ such that $f=\lambda h+$ $f_{1}+\cdots+f_{t}$.

Proof. From Lemma 1.1, there is a positive number $\lambda$ such that $f-\lambda h \in$ $\delta$. From the above theorem there exist atomic assignments $f_{1}, \ldots, f_{t}$ such that $f-\lambda h=f_{1}+\cdots+f_{t}$, i.e. $f=\lambda h+f_{1}+\cdots+f_{t}$.

We are now in a position to show that $\mathscr{D}(\mathcal{S})$ is a point lattice.

Corollary 1.2. $\mathscr{D}(\mathcal{D})$ is a point lattice.

Proof. From Theorem 1.1, for any $|f| \in \mathscr{D}(\mathcal{S})$ where $f$ is an assignment in $\delta$, there are atomic assignments $f_{1}, f_{2}, \ldots, f_{t}$ in $\delta$ so that $f=f_{1}+f_{2}$ $+\cdots+f_{t}$. Hence $|f|=\left|f_{1}\right| \cup\left|f_{2}\right| \cup \cdots \cup\left|f_{t}\right|$. But this implies that every element in $\mathscr{D}(\mathcal{S})$ is a join of atoms in $\mathscr{D}(\mathcal{S})$, i.e. $\mathscr{D}(\mathcal{S})$ is a point semilattice.

To see that $\mathscr{D}(\mathcal{S})$ is a lattice, pick $|f|$ and $|h|$ in $\mathscr{D}(\mathcal{S})$. Let $S=$ atoms $\left|f_{k}\right|$ so that $\left|f_{k}\right| \subseteq|f|$ and $\left.\left|f_{k}\right| \subseteq|h|, k=1,2, \ldots, t\right\}$. If $S=\varnothing$, then define $|f| \wedge|h|=\varnothing$. If $S \neq \varnothing$, then define $|f| \wedge|h|=\cup_{k=1}^{t}\left|f_{k}\right|$. Thus, $\mathscr{D}(\delta)$ is a lattice.

In fact, this lattice possesses the further property that for each assignment $f \in \mathcal{S},[\varnothing,|f|]$ is complemented. We call lattices with this property ideal complemented lattices.

THEOREM 1.2. If $|f| \in \mathscr{D}(\mathcal{S})$ then for each $|h| \in \mathscr{D}(\mathcal{S})$ with $|h| \subseteq|f|$ there exists $|\bar{h}| \in \mathscr{D}(\delta)$ such that $|h| \cup|\bar{h}|=|f|$ and $|h| \wedge|\bar{h}|=\varnothing$.

Proof. If $|f|=\varnothing$ then $|h|=\varnothing$ so we choose $|\bar{h}|=\varnothing$. If $|f| \neq \varnothing$ but $|h|=\varnothing$, choose $|\bar{h}|=|f|$ while if $|h|=|f|$ choose $|\bar{h}|=\varnothing$. Now suppose $\varnothing \subsetneq|h| \subsetneq|f|$.

Let $\bar{h}$ be an assignment so that $|h| \cup|\bar{h}|=|f|$ and if $k$ is an assignment so that $|h| \cup|k|=|f|$ then $\operatorname{card}|\bar{h}| \leqslant \operatorname{card}|k|$. Now suppose $|h| \cap|\bar{h}| \neq \varnothing$. Then there is an assignment $k$ so that $|k| \subseteq|h|$ and $|k| \subseteq|\bar{h}|$. Pick a scalar $\lambda$ so that $\bar{h}-\lambda k$ is an assignment and $|\bar{h}-\lambda k| \subsetneq|\bar{h}|$. Then $|h| \cup|\bar{h}-\lambda k|=|f|$ and $\operatorname{card}|\bar{h}-\lambda k|<\operatorname{card}|\bar{h}|$. This yields a contradiction and hence $|h| \cap|\bar{h}|=\varnothing$.

This theorem then provides a useful decomposition property for patterns in $\mathcal{D}(\delta)$. In particular, it follows that if $K \in \mathcal{D}(\mathcal{S})$ is not an atom then there are $K_{1}$ and $K_{2}$ in $\mathscr{D}(\delta)$ with card $K_{1}<$ card $K$ and card $K_{2}<$ card $K$ so that $K_{1} \cup K_{2}=K$ and $K_{1} \wedge K_{2}=\varnothing$. Decomposition results of this type are very useful, as tools in inductive arguments, in investigating the structure of patterns in $\mathscr{D}(\mathcal{S})$.

As a final structural result on $\mathscr{D}(\mathcal{S})$, based on the above techniques, we add the following.

Corollary 1.3. Suppose $|f| \in \mathscr{D}(\mathcal{S})$. Then $|f|$ covers at most two atoms. 
Proof. Assume to the contrary that $|f|$ covers atoms $\left|f_{1}\right|, \ldots,\left|f_{t}\right|, t>3$. Thus $\left|f_{1}+f_{2}\right|=\left|f_{2}+f_{3}\right|=|f|$ and so by Lemma 1.1 , there is a positive number $\lambda$ such that $f_{1}+f_{2}-\lambda\left(f_{2}+f_{3}\right) \in \mathcal{S}$ with $\left|f_{1}+f_{2}-\lambda\left(f_{2}+f_{3}\right)\right| \subsetneq \mid f_{1}$ $+f_{2}|=| f \mid$. Suppose $\left|f_{1}+f_{2}-\lambda\left(f_{2}+f_{3}\right)\right|=\varnothing$. Then $f_{1}+f_{2}=\lambda f_{2}+\lambda f_{3}$. If $\lambda=1,\left|f_{1}\right|=\left|f_{3}\right|$, a contradiction. If $\lambda>1, f_{1}=(\lambda-1) f_{2}+\lambda f_{3}$ and $\left|f_{1}\right|=$ $\left|f_{2}\right| \cup\left|f_{3}\right|$, again a contradiction. The case $\lambda<1$ is similar. From this, we conclude that $f_{1}+f_{2}-\lambda\left(f_{2}+f_{3}\right)=h \neq 0$, where $|h| \in A=\left\{\left|f_{1}\right|, \ldots,\left|f_{t}\right|\right\}$. By Lemma 1.2, $h=\lambda_{0} f_{k}, \lambda_{0}$ a positive number. Again from Lemma 1.1, there exist positive numbers $\lambda_{1}, \lambda_{2}$ such that $f_{1}+f_{2}-\lambda_{1} \lambda_{0} f_{k_{0}} \in \mathcal{S}$, $\left|f_{1}+f_{2}-\lambda_{1} \lambda_{0} f_{k}\right| \subset\left|f_{1}+f_{2}\right|$ and $f_{2}+f_{3}-\lambda_{2} \lambda_{0} f_{k} \in \mathcal{S}$ with $\left|f_{2}+f_{3}-\lambda_{2} \lambda_{0} f_{k}\right| \subsetneq\left|f_{2}+f_{3}\right|$. As above, we find that $f_{1}+f_{2} \neq \lambda_{1} \lambda_{0} f_{k}$ and $f_{2}+f_{3} \neq \lambda_{2} \lambda_{0} f_{k}$. Consequently, there are positive numbers $\lambda_{3}, \lambda_{4}, f_{i}, f_{j} \in A$ such that $f_{1}+f_{2}-\lambda_{1} \lambda_{0} f_{k}=\lambda_{3} f_{i}$ and $f_{2}+f_{3}-\lambda_{2} \lambda_{0} f_{k}=\lambda_{4} f_{j}$. From this we obtain $\left(\lambda_{0}+\lambda \lambda_{2} \lambda_{0}-\lambda_{1} \lambda_{0}\right) f_{k}+\lambda \lambda_{4} f_{j}=\lambda_{3} f_{i}$. This again gives a contradiction and the result is now established.

This then completes our initial study of the structure of $\mathscr{D}(\delta)$. In the next section we develop an investigative tool, called subspace transformations, to be used in furthering the study of the structure of $\mathscr{D}(\mathcal{S})$.

2. Subspace transformation on $\mathcal{S}$. The purpose of this section is to determine a subspace of $V$ which can be used, in an algebraic way, to determine all patterns in $\mathscr{D}(\mathcal{S})$. For this, let $W$ be a subspace of $V$ so that

(i) $W \cap \mathcal{S}=\{0\}$ and

(ii) for each pair of assignments $f$ and $h$ in $\mathcal{S}$, there is a positive number $\lambda$ with $f-\lambda h \in W$.

The existence of such a $W$ is guaranteed by the following lemma.

LEMMA 2.1. For any given $V$ there exists a subspace of $V$ satisfying properties (i) and (ii).

Proof. Let $l=f_{1}+f_{2}+\cdots+f_{m}$ where $f_{1}, f_{2}, \ldots, f_{m}$ is a set of assignments representing the atoms in $\mathscr{D}(\mathcal{S})$. Let $\rho_{x}>0$ for $x \in \mathcal{C}$ and define $W=\left\{k \in V \mid l \circ k=\Sigma_{x \in \Theta^{\circ}} l(x) k(x)=0\right\}$, a subspace of $V$. Now pick any assignment $f \in \mathcal{S}$. Set $l \circ f=r_{1}$ and $l \circ l=r_{2}$. Pick $\lambda>0$ so that $\lambda r_{1}-r_{2}=$ 0 . Consider $\lambda f-l \in V$. Then $l \circ(\lambda f-l)=\lambda l \circ f-l \circ l=\lambda r_{1}-r_{2}=0$ so that $\lambda f-l \in W$. Pick any assignment $h \in \mathcal{S}$. As in the previous case, there is a $\beta>0$ so that $\beta h-l \in W$. Hence $(\lambda f-l)-(\beta h-l)=\lambda f-\beta h \in W$ so that $\left(\lambda \beta^{-1}\right) f-h \in W$ from which the lemma follows.

The nonzero functions in $W$ are called chains. As an initial use of these chains we characterize the atomic assignments in $\mathcal{S}$.

LEMMA 2.2. An assignment $f \in \mathcal{S}$ is atomic if and only if there is no chain $g$ in $W$ with $|g| \subseteq|f|$. 
Proof. For the direct implication, let $f$ be an atomic assignment in $\mathcal{S}$. Suppose there is a chain $g$ in $W$ so that $|g| \subseteq|f|$. Then pick $\lambda$ so that $f-\lambda g \in \mathcal{S}$ with $|f-\lambda g| \subsetneq|f|$. Thus, as $f$ is an atomic assignment $f-\lambda g=$ 0 . But now as $\delta \cap W=\{0\}, f=g=0$ a contradiction.

Conversely, suppose $f$ is an assignment which is not atomic. In this case there is an atomic assignment $f_{1} \in \mathcal{S}$ with $\left|f_{1}\right| \subsetneq|f|$. Pick $\lambda$ so that $\lambda f-f_{1} \in$ $W$. Hence, there is a chain $g$ in $W$ so that $\lambda f-f_{1}=g$. Now $|g| \subseteq|f|$ from which the lemma follows.

These chains will now be used to provide transformations on $\mathcal{S}$. This can be accomplished by noticing that if $h$ is an assignment and $f$ is an assignment, there is a positive number $\lambda$ and a $g \in W$ so that $f=\lambda h+g$. We call the transformation of $h$ to $f$ a subspace transformation. Thus, with positive numbers $\lambda$ and $g$ 's in $W$, the cone $\mathcal{S}$ may be transversed by subspace transformations.

It should be noted, however, that the subspace $W$ can be quite large. Hence, we intend to show that $\mathcal{S}$ can be transversed by using only atomic chains in $W$, i.e., those chains in $W$ which are atoms in $\mathscr{D}(W)$. These chains are easily determined by their supports.

LEMma 2.3. Suppose $g_{1}$ and $g_{2}$ are atomic chains so that $\left|g_{1}\right|=\left|g_{2}\right|$. Then there is a number $\lambda$ so that $g_{1}=\lambda g_{2}$.

Proof. Pick any $x \in\left|g_{1}\right|=\left|g_{2}\right|$. Let $\lambda$ be such that $g_{1}(x)=\lambda g_{2}(x)$. Then $g_{1}-\lambda g_{2} \in W$ and $\left|g_{1}-\lambda g_{2}\right| \subsetneq\left|g_{1}\right|$ so that $\left|g_{1}-\lambda g_{2}\right|=\varnothing$. Thus, $g_{1}=\lambda g_{2}$.

The atomic chains in $W$ completely determine $W$.

LEMma 2.4. Suppose $g$ is a chain in $W$. Then there are atomic chains $g_{1}$, $g_{2}, \ldots, g_{t} \in W$ with $\left|g_{i}\right| \subseteq|g|$ for $i=1,2, \ldots, t$ so that $g=g_{1}+g_{2}$ $+\cdots+g_{t}$.

Proof. The proof is by induction on card $|g|$. If card $|g|=$ $\min _{k \text { a chain }}(\operatorname{card}|k|)$, then $g$ is atomic and the theorem holds. Thus, suppose the theorem holds for all chains $g \in W$ with card $|g|<r$. Now pick any chain $g \in W$ with card $|g|=r$. If $g$ is atomic, there is nothing to prove, hence, suppose $g$ is not an atomic chain. In this case there is an atomic chain $\bar{g} \in W$ so that $|\bar{g}| \subsetneq|g|$. Pick any $x \in|\bar{g}|$ and let $\lambda$ be such that $g(x)=$ $\lambda \bar{g}(x)$. Consider the chain $g-\lambda \bar{g} \in W$. As card $|g-\lambda \bar{g}|<r$, by the induction hypothesis there are atomic chains $g_{2}, \ldots, g_{t}$ in $W$ with $\left|g_{i}\right| \subseteq \mid g$ $-\lambda \bar{g}|\subseteq| g \mid$ so that $g-\lambda \bar{g}=g_{2}+\cdots+g_{t}$. Thus, setting $g_{1}=\lambda \bar{g}$ yields $g=g_{1}+g_{2}+\cdots+g_{t}$ the result of the lemma.

To develop the relationship between $W$ and $\mathcal{S}$ requires the introduction of the concept of sign compatibility in $W$. For this, a chain $\bar{g}$ is said to be sign compatible with a chain $g$ if and only if $\bar{g}(x) g(x)>0$ for all $x \in \mathcal{C}$. 
As an initial result on sign compatibility, we give the following.

LEMMA 2.5. Let $g$ and $\bar{g}$ be chains in $W$ with $|\bar{g}| \subseteq|g|$. Then there is a number $\lambda$ so that $g-\lambda \bar{g}$ is sign compatible with $g$ and $|g-\lambda \bar{g}| \subsetneq|g|$.

Proof. Set $|\bar{g}|=\left\{x_{1}, x_{2}, \ldots, x_{t}\right\}$. Define $\lambda_{k}=g\left(x_{k}\right) / \bar{g}\left(x_{k}\right)$ for $k=$ $1,2, \ldots, t$. Let $\left|\lambda_{k_{0}}\right|=\min _{1<k<t}\left|\lambda_{k}\right|$. Then $g-\lambda_{k_{0}} \bar{g} \in W$ with $\mid g-$ $\lambda_{k_{0}} \bar{g}|\subsetneq| g \mid$.

That $g-\lambda_{k_{0}} g$ is sign compatible with $g$ is argued by contradiction. For this, assume there is an $x \in|\bar{g}|$ with $g(x)>0$ and $\left(g-\lambda_{k_{0}} \bar{g}\right)(x)<0$. But now $g(x)<\lambda_{k_{0}} \bar{g}(x)$ so that $g(x) / \bar{g}(x)<\lambda_{k_{0}}$ which contradicts $\left|\lambda_{k_{0}}\right|=$ $\min _{1<k<t}\left|\lambda_{k}\right|$. Hence, the lemma follows.

From this lemma, a major result, required in relating $W$ and $\mathcal{S}$, can be given.

THEOREM 2.1. Let $g$ be a chain in $W$ with $x \in|g|$. Then there is an atomic chain $\bar{g} \in W$, sign compatible with $g$, so that $x \in|\bar{g}| \subseteq|g|$.

Proof. If possible, pick an atomic $g_{1} \in W$ so that $x \notin\left|g_{1}\right| \subseteq|g|$. By Lemma 2.5, let $\lambda_{1}$ be a number so that $\left|g-\lambda_{1} g_{1}\right| \subsetneq|g|$ and $g-\lambda_{1} g_{1}$ sign compatible with $g$.

By replacing $g$ by $g-\lambda_{1} g_{1}$, the above procedure may be repeated, and in fact repeated until a chain $\bar{g} \in W$ is found so that $x \in|\bar{g}| \subseteq|g|$ with $\bar{g}$ sign compatible with $g$ so that if $\bar{g}_{1}$ is an atomic chain in $W$ with $\left|\bar{g}_{1}\right| \subseteq|\bar{g}|$, then $x \in\left|\bar{g}_{1}\right|$. If possible, pick any such $\bar{g}_{1}$. By Lemma 2.5 , let $\lambda_{2}$ be a number, so that $\left|\bar{g}-\lambda_{2} \bar{g}_{1}\right| \subsetneq|\bar{g}|$ and $\bar{g}-\lambda_{2} \bar{g}_{1}$, sign compatible with $\bar{g}$. Note, if $(\bar{g}-$ $\left.\lambda_{2} \bar{g}_{1}\right)(x)=0$, then $\bar{g}=\lambda_{2} \bar{g}_{1}$ as there is no atomic chain $g_{l} \in W,\left|g_{l}\right| \subseteq|\bar{g}|$ so that $x \notin\left|g_{l}\right|$. Thus, if $\bar{g}$ is not an atomic chain, $x \in\left|\bar{g}-\lambda_{2} \bar{g}_{1}\right|$. From this remark, it is clear that by replacing $\bar{g}$ by $\bar{g}-\lambda_{2} \bar{g}_{1}$, the above procedure may be repeated, and in fact repeated until an atomic chain $\bar{g}_{1} \in W$ is found so that $x \in\left|\bar{g}_{1}\right| \subseteq|\bar{g}| \subseteq|g|$ and sign compatible with $\bar{g}$ and hence, $g$. This then is the result of the theorem.

These results now place us in a position of being able to show that $\mathcal{S}$ may be transversed by chains in $W$. Our initial result in this regard follows.

LEMMA 2.6. Suppose $h$ and $f$ are assignments in $\mathcal{S}$ with $|h| \subseteq|f|$. Then there is a positive number $\lambda$ and a sequence of atomic chains $g_{1}, g_{2}, \ldots, g_{t}$ in $W$ so that $f=\lambda h+g_{1}+\cdots+g_{t}$ with $\lambda h+g_{1}+\cdots+g_{r} \in \mathcal{S}$ for $r=1$, $2, \ldots, t$.

Proof. By the definition of $W$, pick a positive number $\lambda_{0}$ so that $f-\lambda_{0} h=$ $g \in W$. The method of proof is now by induction on card $|g|$. By Theorem 2.1, pick an atomic chain $g_{1}$ in $W$ compatible with $g$ so that $\left|g_{1}\right| \subseteq|g|$. By Lemma 2.5 , pick a number $\lambda_{1}$ so that $g-\lambda_{1} g_{1}$ is sign compatible with $g$ and 
$\left|g-\lambda_{1} \bar{g}_{1}\right| \subsetneq|g|$. Note that $\lambda_{1}>0$ as $\bar{g}_{1}$ is sign compatible with $g$. Now consider $\lambda_{0} \bar{h}+\lambda_{1} \bar{g}_{1} \in W$. We show $\lambda_{0} h+\lambda_{1} \bar{g}_{1} \in \mathcal{S}$. For this we argue by cases.

Case 1. $x \in|f|-|h|$. As $\bar{g}_{1}$ is sign compatible with $g=f-\lambda_{0} h$, and $g(x)>0$ it follows that $\bar{g}_{1}(x) \geqslant 0$ and so $\left(\lambda_{0} h+\lambda_{1} \bar{g}_{1}\right)(x)>0$.

Case 2. $x \in|h|$. First note that if $\bar{g}_{1}(x) \geqslant 0$, then $\left(\lambda_{0} h+\lambda_{1} g_{1}\right)(x)>0$, thus, we argue for $\bar{g}_{1}(x)<0$. In this case, as $\bar{g}_{1}$ is sign compatible with $g=f-$ $\lambda_{0} h, g(x)<0$ and hence, $0<f(x)<\lambda_{0} h(x)$. Further, as $g-\lambda_{1} \bar{g}_{1}$ is sign compatible with $g,\left(g-\lambda_{1} \bar{g}_{1}\right)(x)<0$, i.e. $g(x)<\lambda_{1} \bar{g}_{1}(x)<0$. Thus, $0<$ $f(x)=\lambda_{0} h(x)+g(x) \leqslant \lambda_{0} h(x)+\lambda_{1} \bar{g}_{1}(x)$. Hence, $\lambda_{0} h+\lambda_{1} \bar{g}_{1} \in \delta$.

Replacing $h$ by $\lambda_{0} h+\lambda_{1} \bar{g}_{1}$ and applying the induction hypothesis assures the existence of positive number $\lambda_{2}$ and atomic chains $g_{1}, \ldots, g_{t}$ in $W$ so that $f=\lambda_{2}\left(\lambda_{0} h+\lambda_{1} \bar{g}_{1}\right)+g_{2}+\cdots+g_{t}$ with $\lambda_{2}\left(\lambda_{0} h+\lambda_{1} \bar{g}_{1}\right)+g_{2}+\cdots+g_{r}$ $\in \mathcal{S}$ for $r=2, \ldots, t$. Thus, setting $\lambda=\lambda_{2} \lambda_{0}$, and $g_{1}=\lambda_{2} \lambda_{1} \bar{g}_{1}$, the lemma follows.

Based on this lemma, the desired result on transversing $\delta$ by using chains can now be given.

THEOREM 2.2. Suppose $f$ and $h$ are assignments in $\delta$. Then there is a positive number $\lambda$ and $a$ sequence of atomic chains $g_{1}, g_{2}, \ldots, g_{t}$ in $W$ so that $f=\lambda h+g_{1}+g_{2}+\cdots+g_{t}$ with $\lambda h+g_{1}+\cdots+g_{r} \in \mathcal{S}$ for $r=1$, $2, \ldots, t$.

Proof. Let $k=f+h$. Then $|f| \subseteq|k|$ and $|h| \subseteq|k|$. Thus by Lemma 2.6, there are positive numbers $\lambda_{1}$ and $\lambda_{2}$ with sequences of atomic chains $g_{1}$, $g_{2}, \ldots, g_{r}$ and $\bar{g}_{1}, \bar{g}_{2}, \ldots, \bar{g}_{t}$ so that $k=\lambda_{1} h+g_{1}+\cdots+g_{r}$ with $\lambda_{1} h+g_{1}$ $+\cdots+g_{k} \in \mathcal{S}$ for $k=1,2, \ldots, r$ and $k=\lambda_{2} f+\bar{g}_{1}+\cdots+\bar{g}_{t}$ with $\lambda_{2} f+\bar{g}_{1}+\cdots+\bar{g}_{k} \in \delta$ for $k=1,2, \ldots, t$. Now $k-\bar{g}_{t}-\cdots-\bar{g}_{t-k}$ for $k=0,1, \ldots, t-1$ and hence, $\lambda_{1} h+g_{1}+\cdots+g_{r}-\bar{g}_{t}-\cdots-$ $\bar{g}_{t-k} \in \mathcal{S}$ for $k=0,1, \ldots, t-1$, with $\lambda_{1} h+g_{1}+\cdots+g_{r}-\bar{g}_{t}-\cdots-$ $\bar{g}_{1}=\lambda_{2} f$. Dividing both sides of this equation by $\lambda_{2}$ then yields the result.

Thus, from this theorem $\delta$ can be transversed by using subspace transformations with atomic chains.

Concerning $\mathscr{D}(\mathcal{S})$ we have the following.

THEOREM 2.3. Suppose $f$ is an assignment. If $h$ is any other assignment then there is a chain $g$ so that $|f|=|h+g|$.

Further, local moves in $\mathscr{D}(\delta)$ may be obtained through the use of only one atomic chain.

COROLLARY 2.1. Suppose $f$ and $h$ are assignments in $\delta$ such that $|f|$ covers $|h|$ in $\mathscr{D}(\delta)$. Then there is an atomic chain $g$ in $W$ such that $|h+g|=|f|$. Similarly, there is an atomic chain $g$ in $W$ such that $|h|=|f+g|$. 
Proof. Let $\lambda$ be a positive number so that $f-\lambda h=g_{1} \in W$. Thus, $f=\lambda h+g_{1}$. Pick $x \in|f|-|h|$. By Theorem 2.1, let $g_{2}$ be an atomic chain in $W$ sign compatible with $g_{1}$ so that $g_{2}(x) \neq 0$. Consider $h+\beta g_{2}$. For sufficiently small $\beta>0, h+\beta g_{2} \in \mathcal{S}$. Now $|h| \subset\left|h+\beta g_{2}\right| \subseteq\left|h+g_{1}\right|=|f|$ so that $\left|h+\beta g_{2}\right|=|f|$. The first part of the corollary now follows by setting $\boldsymbol{g}=\beta g_{2}$.

The proof of the second part of the corollary follows similarly.

From the above results, it is seen that any assignment in $\mathcal{S}$ can be manipulated into any other assignment in $\delta$ by applying subspace transformations. Thus, given any pattern in $\mathscr{D}(\mathcal{S})$, all other patterns in $\mathscr{D}(\mathcal{S})$ are completely determined by $W$.

This then concludes the development of subspace transformations. Further application of this work will be found in the next section where we determine some of the local structure of $\mathscr{D}(\mathcal{S})$.

3. The local structure of $\mathscr{D}(\delta)$. The global structure of $\mathscr{D}(\delta)$ seems complex. However, in this section, we can give a description of some local structure of $\mathscr{D}(\mathcal{\delta})$. The work uses as an investigative tool the notion of a cluster. For this, if $K \in \mathscr{D}(\delta)$ define

$$
\operatorname{cl}(K)=\{x \in \mathcal{C} \mid K+x \in \mathscr{D}(\mathcal{S})\}
$$

It is easy to see that $K \subseteq \operatorname{cl}(K)$. Further, we can show that $\operatorname{cl}(K)$, as well as every set between $K$ and $\operatorname{cl}(K)$, is in $\mathscr{D}(\mathcal{S})$.

LEMMA 3.1. If $K \in \mathscr{D}(\delta)$ and $S \subseteq \operatorname{cl}(K)$, then $K+S \in \mathscr{D}(\delta)$.

Proof. Let $f$ be an assignment in $\mathcal{S}$ so that $|f|=K$. For each $x \in S$, let $f_{x}$ be an assignment in $\mathcal{S}$ so that $\left|f_{x}\right|=K+x$. Thus, $f+\Sigma_{x \in S} f_{x}$ is an assignment in $\mathcal{S}$ and $\left|f+\Sigma_{x \in S} f_{x}\right|=K+S$ from which the lemma follows.

Thus, $\mathrm{cl}(\cdot)$ is a mapping from $\mathscr{D}(\mathcal{S})$ into $\mathscr{D}(\mathcal{S})$. This mapping is in fact idempotent and hence, a closure mapping.

TheOREM 3.1. If $K \in \mathscr{D}(\delta)$, then $\operatorname{cl}[\operatorname{cl}(K)]=\operatorname{cl}(K)$.

Proof. Of course, $\operatorname{cl}(K) \subseteq \operatorname{cl}[\operatorname{cl}(K)]$. Further, if $\operatorname{cl}[\operatorname{cl}(K)] \subseteq \operatorname{cl}(K)$ there is nothing to prove, thus suppose there is an $x \in \operatorname{cl}[\operatorname{cl}(K)]-\operatorname{cl}(K)$. Pick $S=$ $\left\{x_{1}, \ldots, x_{d}\right\} \subseteq \operatorname{cl}(K)-K$ so that $K+S+x \in \mathcal{D}(\delta)$. The proof now follows by induction on $d$. If $d=0, K+x \in \mathscr{D}(\mathcal{S})$ so that $x \in \operatorname{cl}(K)$ a contradiction. Now suppose that for all $d, 0 \leqslant d<d_{1}$, a contradiction is obtained. Suppose now that $d=d_{1}$.

Let $f$ be an assignment in $\mathcal{S}$ so that $|f|=K$. For each $k, 1 \leqslant k<d$ pick a chain $g_{k} \in W$ so that $f+g_{k} \in \mathcal{S},\left|f+g_{k}\right|=K+x_{k}$ and $h=f+g_{1}$ 
$+\cdots+g_{d} \in \mathcal{S}$. Let $g$ be a chain in $W$ where $h+g \in \mathcal{S}$ and $|h+g|=K$ $+S+x$. We now argue by cases.

Case 1. For some $k, x_{k} \notin|g|$.

Without loss of generality, suppose $k=d$. In this case for $\lambda$ sufficiently large, $\lambda f+g_{1}+\cdots+g_{d-1} \in \delta$ and for $\beta$ sufficiently small $\lambda f+g_{1}$ $+\cdots+g_{d-1}+\beta g \in \delta$. Hence $\left|\lambda f+g_{1}+\cdots+g_{d-1}\right|=K+\left(S-x_{d}\right)$, $\left|\lambda f+g_{1}+\cdots+g_{d-1}+\beta g\right|=K+\left(S-x_{d}\right)+x$. Thus, by the induction hypothesis, a contradiction can be obtained.

Case 2. Each $x_{k} \in|g|$.

As $x_{d} \in|g| \cap\left|g_{d}\right|$, take $\gamma$ so that $\left(g-\gamma g_{d}\right)\left(x_{d}\right)=0$. Now for $\lambda$ sufficiently large, $\lambda f+g_{1}+\cdots+g_{d-1} \in \mathcal{S}$ and for $\beta$ sufficiently small, $\lambda f+g_{1}$ $+\cdots+g_{d-1}+\beta\left(g-\gamma g_{d}\right) \in$ S. Now $\left|\lambda f+g_{1}+\cdots+g_{d-1}\right|=K+(S$ $\left.-x_{d}\right)$ and $\left|\lambda f+g_{1}+\cdots+g_{d-1}+\beta\left(g-\gamma g_{d}\right)\right|=K+\left(S-x_{d}\right)+x$ and hence, by the induction hypothesis, a contradiction can be obtained.

From this, the theorem follows.

As a consequence of this theorem, we have the following characterization.

Corollary 3.1. Let $K \in \mathscr{D}(\delta)$. Then $K=\mathrm{cl}(K)$ if and only if for each atomic chain $g, \operatorname{card}(|g|-K) \neq 1$.

Thus, $\operatorname{cl}(K)-K$ gives an upper bound on the set $T$ so that $K+S \in$ $\mathscr{D}(\delta)$ for each $S \subseteq T$. Considering now a lower bound on $K$ so that $K+S \in \mathscr{D}(\delta)$ for all $S \subseteq T$ motivates the following special set.

If $K \in \mathscr{D}(\delta)$ and $K-x \notin \mathscr{D}(\delta)$ for each $x \in K$, we call $K$ minimal. Thus, a minimal pattern $K$ has the property that every set between $K$ and $\operatorname{cl}(K)$ is in $\mathscr{D}(\mathcal{S})$. Further, this is the largest interval containing $K$, in $\mathscr{D}(\mathcal{S})$ with this property. This then describes some of the local structure of $\mathscr{D}(\delta)$.

It is also of interest that these intervals provide a covering of $\mathscr{D}(\mathcal{S})$.

LEMma 3.2. If $S \in \mathscr{D}(\mathcal{S})$, then there is a minimal $K \in \mathscr{D}(\delta)$ so that $S \in[K, \mathrm{cl}(K)]$.

Proof. The proof is by induction on $d=$ card $S$. If $S$ is minimal, there is nothing to prove. Thus, suppose $S$ is not minimal. Then there is an $x \in S$ so that $S-x \in \mathscr{D}(\mathcal{S})$. By the induction hypothesis, there is a minimal $K$ so that $S-x \in[K, \operatorname{cl}(K)]$. As $\operatorname{cl}[\mathrm{cl}(K)]=\operatorname{cl}(K), S \in[K, \operatorname{cl}(K)]$ from which the lemma follows.

From this lemma then, it is seen that if the minimal patterns and their clusters in $\mathscr{D}(\delta)$ can be established, then all of $\mathscr{D}(\delta)$ is determined. Thus, any structure which we can determine about these patterns is also of some importance. Hence, we shall conclude this section by considering the structure of these sets.

Concerning the minimal patterns, examples are easily constructed which 
show that if $K_{1}$ and $K_{2}$ are minimal in $\mathscr{D}(\mathcal{S}), K_{1} \cup K_{2}$ need not be minimal. However, $K_{1} \wedge K_{2}$ is minimal. In fact, we can give a stronger result.

LEMMA 3.3. If $K_{1} \in \mathscr{D}(\mathcal{S})$ is minimal and $K_{2} \subseteq K_{1}$ with $K_{2} \in \mathscr{D}(\mathcal{S})$, then $K_{2}$ is minimal.

Proof. The proof is by contradiction. For this, suppose $K_{2}$ is not minimal. In this case, there is an $x \in K_{2}$ with $K_{2}-x \in \mathscr{D}(\mathcal{\delta})$. Pick an assignment $f_{K_{2}-x} \in \mathcal{S}$ so that $\left|f_{K_{2}-x}\right|=K_{2}-x, f_{x} \in \mathcal{S}$ so that $x \in\left|f_{x}\right| \subseteq K_{2}$, and $f_{K_{1}} \in$ $\mathcal{S}$ so that $\left|f_{K_{1}}\right|=K_{1}$. Now consider $\lambda f_{K_{2}-x}+f_{K_{1}}-\beta f_{x}$ where $\beta$ is chosen so that $f_{K_{1}}(x)-\beta f_{x}(x)=0$. Thus, for $\lambda$ sufficiently large, as $\left|f_{x}\right| \subseteq K_{2}, \lambda f_{K_{2}-x}+$ $f_{K_{1}}-\beta f_{x} \in \mathcal{S}$ and $\left|\lambda f_{K_{2}-x}+f_{K_{1}}-\beta f_{x}\right|=K_{1}-x$ which contradicts $K_{1}$ being minimal. Thus, from the contradiction, it follows that $K_{2}$ is minimal.

Similarly, if $K_{1}$ and $K_{2}$ are in $\mathscr{D}(\mathcal{S})$ then $\operatorname{cl}\left(K_{1}\right) \cup \operatorname{cl}\left(K_{2}\right)$ need not be $\operatorname{cl}\left(K_{3}\right)$ for some pattern $K_{3} \in \mathscr{D}(\mathcal{S})$. However, the following property on the meet can be established.

LEMMA 3.4. If $K_{1}$ and $K_{2}$ are in $\mathscr{D}(\mathcal{\delta})$ then $\operatorname{cl}\left[\operatorname{cl}\left(K_{1}\right) \wedge \operatorname{cl}\left(K_{2}\right)\right]=\operatorname{cl}\left(K_{1}\right) \wedge$ $\operatorname{cl}\left(K_{2}\right)$.

Thus, we have that both minimal patterns and clusters form meet semilattices in $\mathscr{D}(\mathcal{S})$.

This then will complete our study of the structure of $\mathscr{D}(\mathcal{S})$. In the next section we provide some combinatorial structures which provide examples of this type of lattice. Further we show how our general theorems apply to these special combinatorial structures.

4. Some examples and applications of $\delta$ and hence $\mathscr{D}(\mathcal{S})$. The purpose of this section is to provide some examples of $\mathcal{S}$, and hence $\mathscr{D}(\mathcal{S})$, which indicated that the set of patterns $\mathscr{D}(\delta)$ is not contrived but in fact gives a unified approach to studying some well-known combinatorial structures. Further, we show how our general theorems can be applied to these combinatorial structures.

We first present a general construction which when applied to particular problems gives several well-known examples of $\mathcal{S}$ and $\mathscr{D}(\mathcal{S})$. For this let $M_{1}$, $M_{2}, \ldots, M_{r}$ be subsets of $\mathcal{C}$, each $x \in \mathcal{C}$ in at least one $M_{k}$ for some $k$. For each $f \in[\mathcal{C}, R]$, define $\sigma_{k}(f)=\Sigma_{x \in M_{k}} f(x), k=1,2, \ldots, r$. Pick $\lambda_{i j}>0$ for $i, j \in\{1,2, \ldots, r\}$. Let $V=\left\{f \in[\mathcal{C}, R] \mid \sigma_{i}(f)=\lambda_{i j} \sigma_{j}(f)\right.$ for all $i$ and $\left.j\right\}$. If $\mathcal{S} \neq\{0\}$, then $\sigma_{k}(f)=r_{k}>0$ for some $k$ and hence for all $k$. Further, $\lambda_{i j}=r_{i} r_{j}^{-1}$.

Define $W=\left\{f \in V \mid \sigma_{k}(f)=0\right.$ for all $\left.k\right\}$. Pick $h \neq 0$ and $f \neq 0$ in $\mathcal{S}$. Then $\sigma_{k}(h)=r_{k}>0$ and $\sigma_{k}(f)=\bar{r}_{k}>0$ for all $k$. Now $\lambda_{i j}=r_{i} r_{j}^{-1}=\bar{r}_{i} \bar{r}_{j}^{-1}$, i.e., $r_{i} \bar{r}_{j}=\bar{r}_{i} r_{j}$. Consider $\bar{r}_{1} h-r_{1} f \in V$. Then 


$$
\sigma_{k}\left(\bar{r}_{1} h-r_{1} f\right)=\bar{r}_{1} \sigma_{k}(h)-r_{1} \sigma_{k}(f)=\bar{r}_{1} r_{k}-r_{1} \bar{r}_{k}=0
$$

and hence,

$$
\bar{r}_{1} h=r_{1} f+\left(\bar{r}_{1} h-r_{1} f\right) \text { or } h=\bar{r}_{1}^{-1} r_{1} f+\bar{r}^{-1}\left(\bar{r}_{1} h-r_{1} f\right)
$$

so that $W$ is a transformation subspace for $\mathscr{D}(\delta)$.

A special type of this example, which is often encountered, possesses two further properties.

(i) Each $x \in \mathcal{C}$ is in at most two of the $M_{k}$ 's.

(ii) If $M_{i_{1}} \cap M_{i_{2}} \neq \varnothing, M_{i_{2}} \cap M_{i_{3}} \neq \varnothing, \ldots, M_{i_{1-1}} \cap M_{i_{1}} \neq \varnothing, M_{i_{1}} \cap M_{i_{1}} \neq$ $\varnothing$, then $t$ is an even integer.

Property (ii) may be realized by considering the graph with vertices $M_{1}, M_{2}, \ldots, M_{r}$ where there is an edge between $M_{i}$ and $M_{j}$ if and only if $M_{i} \cap M_{j} \neq \varnothing$. In terms of this graph, property (ii) may be characterized by saying that all of its cycles have even length.

This general type of example also has a special feature about the atomic chains in $W$.

THEOREM 4.1. If $g$ is an atomic chain in $W$ then $g$ has nonzero values of the same modulus.

Proof. Suppose $g \in W$ is an atomic chain. Let $\mathcal{E}$ denote the graph having vertices $M_{1}, M_{2}, \ldots, M_{r}$ where there is an edge between $M_{i}$ and $M_{j}$ if and only if $\left(M_{i} \cap M_{j}\right) \cap|g| \neq \varnothing$, i.e., $M_{i} \cap M_{j}$ contains an element in the support of $|g|$. We now argue by cases.

Case $1 . \mathcal{E}$ contains a cycle.

For this case, there is a sequence $\left(M_{i_{1}} \cap M_{i_{2}}\right) \cap|g| \neq \varnothing,\left(M_{i_{2}} \cap M_{i_{3}}\right) \cap|g|$ $\neq \varnothing, \ldots,\left(M_{i_{t-1}} \cap M_{i_{i}}\right) \cap|g| \neq \varnothing,\left(M_{i_{i}} \cap M_{i_{1}}\right) \cap|g| \neq \varnothing$ with $t$ an even integer. Pick $x_{k} \in\left(M_{i_{k}} \cap M_{i_{k+1}}\right) \cap|g|(k \bmod t)$. Note that $x_{1}, x_{2}, \ldots, x_{t}$ are distinct elements in $|g|$. Define

$$
e(x)= \begin{cases}1 & \text { if } x=x_{k} \text { and } k \text { is even, } \\ -1 & \text { if } x=x_{k} \text { and } k \text { is odd, } \\ 0 & \text { otherwise }\end{cases}
$$

By direct calculation, $e \in W$. Now, as $\varnothing \neq|e| \subseteq|g|$ and $g$ is atomic it follows that $g=\lambda e$ for some scalar $\lambda$ and hence, the theorem.

Case $2 . \mathcal{E}$ does not contain a cycle.

Pick $x_{1} \in|g|$. Then $x_{1} \in M_{i_{1}}$ for some $i_{1}$, as $-g \in W$ we may assume without loss of generality that $g\left(x_{1}\right)>0$. Then, by the definition of $W$, there exists an $x_{2} \in M_{i_{1}}$ so that $g\left(x_{2}\right)<0$. If $x_{2} \in M_{i_{2}}$ for some $i_{2} \neq i_{1}$, then again by the definition of $W$, there is an $x_{3} \in M_{i_{2}}$ so that $g\left(x_{3}\right)>0$. By continuing this procedure, as $\mathscr{E}$ has no cycles, we obtain a sequence $x_{1}, x_{2}, \ldots, x_{q}$ so that 
(a) $g\left(x_{k}\right)>0$ for $k$ odd, $g\left(x_{k}\right)<0$ for $k$ even, and

(b) $x_{k}, x_{k+1} \in M_{i_{k}}$ for all $k$.

Now if $x_{1} \in M_{j_{1}}$ for some $j_{1} \neq i_{1}$, then by the definition of $W$ there is an $x_{-2} \in M_{j_{1}}$ so that $g\left(x_{-2}\right)<0$. Reproducing the above procedure now generates a sequence $x_{1}, x_{-2}, x_{-3}, \ldots, x_{-p}$ so that

(a) $g\left(x_{-k}\right)>0$ for $k$ odd, $g\left(x_{-k}\right)<0$ for $k$ even, and

(b) $x_{-k}, x_{-(k+1)} \in M_{j_{k}}$ for all $k \geqslant 2$.

Now define

$$
e(x)= \begin{cases}1 & \text { if } x=x_{k} \text { and } k \text { is odd } \\ -1 & \text { if } x=x_{k} \text { and } k \text { is even, } \\ 1 & \text { if } x=x_{-k} \text { and } k \geqslant 2 \text { is odd } \\ -1 & \text { if } x=x_{-k} \text { and } k \geqslant 2 \text { is even } \\ 0 & \text { otherwise. }\end{cases}
$$

By direct calculation, $e \in W$. As $\varnothing \neq|e| \subseteq|g|$ and $g$ is atomic, there is a scalar $\lambda$ so that $g=\lambda e$ from which the theorem follows.

The general construction procedure is now applied in providing several well-known examples of $\delta$ and $\mathscr{D}(\delta)$.

EXAMPLE 4.1. The set of $m \times n$ nonnegative matrices with specified positive row sums a scalar multiple of $r_{1}, r_{2}, \ldots, r_{m}$.

Let $C=\{(i, j)$ where $1 \leqslant i \leqslant m$ and $1 \leqslant j \leqslant n\}$. Let $M_{k}=\{(k, j)$ where $1 \leqslant j \leqslant n\}$ for $1 \leqslant k \leqslant m$. Let $\lambda_{i j}=r_{i} r_{j}^{-1}$ and $V=\left\{f \in[\mathcal{C}, R] \mid \sigma_{i}(f)=\right.$ $\left.\lambda_{i j} \sigma_{j}(f)\right\}$. Then $\mathcal{S}=\{f \in V \mid f(x) \geqslant 0$ for all $x \in \mathcal{C}\}$, when realized as a set of $m \times n$ matrices, is the set of $m \times n$ nonnegative matrices with row sums a scalar multiple of $r_{1}, r_{2}, \ldots, r_{m}$. Thus, $\mathscr{D}(\delta)$ represents the patterns of these matrices.

Note that in this example each atomic chain $g \in W$ has nonzero values of the same modulus. It should also be noted that the generalized stochastic matrices form an example of this class of matrices.

EXAMPLE 4.2. The set of $m \times n$ nonnegative matrices with specified positive row sums and positive column sums a scalar multiple of $r_{1}, \ldots, r_{m}$ and $r_{m+1}, \ldots, r_{m+n}$ respectively.

Let $\mathcal{C}=\{(i, j)$ where $1 \leqslant i \leqslant m$ and $1 \leqslant j \leqslant n\}$. Let $M_{k}=\{(k, j)$ where $1<j<n\}$ for $1<k<m$ and $M_{k}=\{(i, k)$ where $1<i<m\}$ for $m+1<$ $k<m+n$. Let $\lambda_{i j}=r_{i} r_{j}^{-1}$ and $V=\left\{f \in[\mathcal{C}, R] \mid \sigma_{i}(f)=\lambda_{i j} \sigma_{j}(f)\right\}$. Then $\delta$, when realized as a set of $m \times n$ matrices, is the set of $m \times n$ matrices with row sums and column sums a scalar multiple of $r_{1}, \ldots, r_{m}$ and $r_{m+1}, \ldots, r_{m+n}$ respectively. Thus, $\mathscr{D}(\mathcal{S})$ represents the patterns of these matrices.

Again note that each atomic chain $g \in W$ has nonzero values of the same 
modulus. Also it should be noted that the doubly stochastic matrices form an example of this class of matrices.

EXAMPLE 4.3. The set of $n \times n$ nonnegative matrices with equal row sums and trace.

Let $\mathcal{C}=\{(i, j)$ where $1 \leqslant i \leqslant n$ and $1 \leqslant j \leqslant n\}$. Let $M_{k}=\{(k, j)$ where $1 \leqslant j \leqslant n\}$ for $1 \leqslant k<n$. Let $M_{n+1}=\{(i, i)$ where $1 \leqslant i \leqslant n\}$. Let $\lambda_{i j}=1$ and $V=\left\{f \in[\mathcal{C}, R] \mid \sigma_{i}(f)=\lambda_{i j} \sigma_{j}(f)\right\}$. Then $\delta$, when realized as a set of $n \times n$ matrices, is the set of $n \times n$ matrices with row sums and trace all equal, and $\mathscr{D}(\mathcal{S})$ represents the patterns of these matrices.

EXAMPLE 4.4. Fully indecomposable matrices.

Consider the special case of Example 4.2 in which $m=n$ and $r_{k}=1$ for all $k$. It is shown in [2] that $|f| \in \mathscr{D}(\mathcal{S})$ if and only if the matrix realization of $f$ is a direct sum of fully indecomposable matrices. Thus, $\mathscr{D}(\delta)$ gives the set of all patterns of this class of matrices.

EXAMPLE 4.5. Strongly connected digraphs.

Let $\mathcal{C}=\{(i, j)$ where $1 \leqslant i \leqslant n$ and $1 \leqslant j \leqslant n\}$ and $V=\{f \in$ $[\mathcal{C}, R] \mid \Sigma_{k \neq i} f(i, k)=\Sigma_{k \neq i} f(k, i)$ for $\left.1 \leqslant i \leqslant n\right\}$. Let $W=\left\{g \in V \mid \Sigma_{i,}\right.$ $g(i, j)=0\}$. Pick $f \neq 0, h \neq 0$ in $\mathcal{S}$. Let $\lambda$ denote the scalar so that $\Sigma_{i, j} f(i, j)$ $=\lambda \Sigma_{i, j} h(i, j)$. Then, $f-\lambda h \in W$.

Let

$$
g(x)= \begin{cases}-1 & \text { if } x=(1,2) \text { and } x=(2,1) \\ 2 & \text { if } x=(1,1) \\ 0 & \text { otherwise. }\end{cases}
$$

Then $g$ is an atomic chain in $W$ yet its nonzero values are not of the same modulus.

Define a digraph $\xi_{f}$ associated with $f \in \mathcal{S}$ as follows. Let $\xi_{f}$ have vertices $v_{1}, v_{2}, \ldots, v_{n}$ and having an arc from $v_{i}$ to $v_{j}$ if and only if $(i, j) \in|f|$. It is shown in [1] that if $f \in \delta, \xi_{f}$ is a disjoint union of strongly connected digraphs. Further, any disjoint union of strongly connected digraphs is some $\xi_{f}$ where $f \in \mathcal{S}$. Thus $\mathscr{D}(\mathcal{S})$ gives the set of all disjoint unions of strongly connected digraphs having $n$ vertices.

Concluding this example, we can also cite that a square nonnegative matrix is irreducible if and only if its associated digraph is strongly connected [1]. Thus, the study of irreducible matrices may be done in this setting.

The above examples have certain properties in common, i.e., in each example the set $C$ is of the form $A \times B$ for some sets $A$ and $B$. In others the atomic chains in $W$ all have nonzero values with the same modulus. We now give examples in which some of these common features are missing.

EXAMPLE 4.6. Another example in which the atomic chains in $W$ do not have nonzero values of the same modulus. 
Let $C=\{(i, j)$ where $1 \leqslant i \leqslant 2$ and $1 \leqslant j \leqslant 2\}$. Let $V=\{f \in$ $[\mathcal{C}, R] \mid f(1,1)+f(1,2)=f(2,1)+f(2,2)$ and $2 f(1,1)+4 f(2,1)=f(1,2)$ $+2 f(2,2)\}$. Note that

$$
f_{1}(x)= \begin{cases}1 / 3 & \text { if } x=(1,1) \\ 1 / 3 & \text { if } x=(2,1) \\ 2 / 3 & \text { if } x=(1,2) \\ 2 / 3 & \text { if } x=(2,2)\end{cases}
$$

and

$$
f_{2}(x)= \begin{cases}1 & \text { if } x=(1,1) \text { and } x=(2,2), \\ 0 & \text { otherwise }\end{cases}
$$

are both in $V$. Further, it can be shown that

$$
g_{1}(x)= \begin{cases}2 & \text { if } x=(1,1) \\ -2 & \text { if } x=(1,2) \\ -1 & \text { if } x=(2,1) \\ 1 & \text { if } x=(2,2)\end{cases}
$$

is an atomic chain in the associated $W$.

Thus, the atomic chains in $W$ do not have nonzero values of the same modulus.

EXAMPLE 4.7. An example in which $\mathcal{C}$ is not of the form $A \times B$ for some $A$ and $B$.

Let $\mathcal{C}=\{1,2, \ldots, n\}$. Then $[\mathcal{C}: R]=\{x \mid x$ is an $n \times 1$ column vector $\}$. Let $A$ be an $m \times n$ real matrix and $b$ an $m \times 1$ nonzero column vector. Now let $V=\{x \mid A x=\lambda b$ for some $\lambda\}$. Then $\mathcal{C}$ is not of the form $A \times B$ for some sets $A$ and $B$.

We now turn our attention to showing how our general theorems can be applied to specific problems. The first problems we consider in this regard concern the structure of special combinatorial systems.

For this, consider a strongly connected digraph $G$ with vertices $V=\left\{v_{1}\right.$, $\left.v_{2}, \ldots, v_{n}\right\}$ and $\operatorname{arcs} A \subseteq V \times V$. Now $\operatorname{cl}(A)=V \times V$. Suppose $G$ is minimally strongly connected. Let $C \subseteq A$ be a circuit in $G$. Since $\mathscr{D}(\delta)$ is an ideal complemented lattice there is a subset $\bar{C} \subseteq A$ in $\mathscr{D}(\delta)$ so that $C \cup \bar{C}$ $=A$ and $C \wedge \bar{C}=\varnothing$. As $\operatorname{cl}(\bar{C}) \neq V \times V$ it follows that $\bar{C}$ is a disjoint union of strongly connected digraphs. By Lemma 3.3, each of these strongly connected digraphs is itself minimally strongly connected. Thus, one sees that any minimally strongly connected digraph is constructed from a disjoint union of minimally strongly connected digraphs which are themselves connected by a circuit. This is illustrated in the following diagram. 


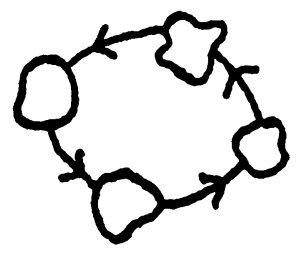

Our general results can also be used to develop special structural results. For example, suppose $G$ is minimally strongly connected with $C \subseteq G$ a circuit in $G$ where card $C=2$. Now, since $\mathscr{D}(\mathcal{S})$ is ideal complemented there is a $\bar{C} \subseteq A$ in $\mathscr{D}(\mathcal{S})$ so that $C \cup \bar{C}=A$ and $\bar{C} \wedge C=\varnothing$. In fact, since $\operatorname{cl}(C) \neq \operatorname{cl}(G), \bar{C} \cap C=\varnothing$. Thus, by Lemma $3.3, \bar{C}$ is a disjoint union of minimally strongly connected digraphs. Since $C$ connects only two vertices, $\bar{C}$ has precisely two components. Hence $G$ is constructed from two disjoint minimally strong digraphs which are connected by $C$ as illustrated in the following diagram.

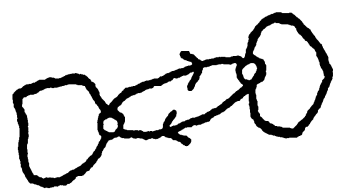

These general results can, of course, also be applied to obtain structural results, similar to those above, for matrix patterns such as those in Examples 4.1 through 4.4.

As a final structural result, we develop a matrix pattern property which, to our knowledge, has not yet been observed. For this, consider the cone $\mathfrak{S}$ generated by the set of nonnegative matrices with fixed row and column sums. Suppose $K$ and $K+S$ are matrix patterns in $\mathscr{D}(\delta)$ with $S \subseteq \operatorname{cl}(K)$. Then, since $\mathrm{cl}(K+S)=\mathrm{cl}(K)$, if $K_{1}$ is a matrix pattern in $\mathscr{D}(\delta)$ with $y \in K_{1}$ and $K_{1}-y \subseteq K+S$, then there is a matrix pattern $K_{2}$ in $\mathscr{D}(\mathcal{S})$ so that $y \in K_{2}$ and $K_{2}-y \subseteq K$. This then yields an interesting property for matrix patterns.

We now consider another type of problem of interest and that is characterizing the edges of cones such as those of $\mathcal{S}$. These vertices are completely determined by their patterns in that they are the atoms of $\mathscr{D}(\mathcal{S})$. These atoms are characterized in Lemma 2.2 in that none of these patterns contains the support of an atomic chain. For the cone generated by the set of nonnegative matrices with specified positive row and column sums it was seen that the supports of the atomic chains could be taken as cycles. Hence, the edges of this cone are precisely those matrices in the cone whose pattern contains no cycle. For the cone generated by the matrices with specified positive row sums, the atomic chains are matrices with precisely two nonzero entries in 
some row and zeros elsewhere. We refer to these atomic chains as shifts. Thus, the edges of this cone are precisely those matrices in the cone whose pattern does not contain the support of any shift.

From this, it should also be seen that the edges of other such cones can also be characterized by using the supports of atomic chains as a tool. In this regard, consider the following example.

Example 4.8. Let $\mathcal{C}=\left\{x_{1}, x_{2}, x_{3}, x_{4}\right\}$ be a set of four distinct points. A function with domain $C$ will be denoted by a vector in $R^{4}$ so that $y=$ $\left(y_{1}, y_{2}, y_{3}, y_{4}\right)^{t}$ denotes the function $y\left(x_{k}\right)=y_{k}$ for $k=1,2,3,4$. Consider $V=\left\{x \mid\left(\begin{array}{lll}1 & 0 & 1\end{array}-1\right) x=0\right\}$. Then the atomic assignments are

$$
\left\{\lambda_{1}\left(\begin{array}{l}
0 \\
1 \\
0 \\
0
\end{array}\right), \lambda_{2}\left(\begin{array}{l}
1 \\
0 \\
0 \\
1
\end{array}\right), \lambda_{3}\left(\begin{array}{l}
0 \\
0 \\
1 \\
1
\end{array}\right] \text { where } \lambda_{1}>0, \lambda_{2}>0 \text { and } \lambda_{3}>0\right\} \text {. }
$$

Let

$$
W=\left\{x \mid\left(\begin{array}{cccc}
1 & 0 & 1 & -1 \\
\frac{1}{2} & 1 & \frac{1}{2} & 1
\end{array}\right) x=0\right\}
$$

and

$$
W^{\prime}=\left\{x \mid\left(\begin{array}{cccc}
1 & 0 & 1 & -1 \\
\frac{1}{3} & 1 & \frac{2}{3} & 1
\end{array}\right) x=0\right\} .
$$

Note that $\left(\begin{array}{lll}1 & 0-10\end{array}\right)^{t} \in W$. Further, as $\left(\left(_{1 / 3}^{1}\right),\left({ }_{2 / 3}^{1}\right)\right.$ is linearly independent, the only $x \in W^{\prime}$ having the form $x=(\alpha 0 \beta 0)^{t}$ is $x=0$. Hence $\{|x| \mid x \in W\} \neq$ $\left\{|x| \mid x \in W^{\prime}\right\}$.

From this we have that different choices of the subspace of atomic chains, say $W$ and $W^{\prime}$, can lead to different sets of supports for these subspaces, i.e., $\bar{W}=\{|x| \mid x \in W\} \neq\left\{|x| \mid x \in W^{\prime}\right\}=\bar{W}^{\prime}$. Hence, this leads to an interesting consequence of our work, namely, that the edges of a cone, such as one of those of the examples, may be characterized by the sets in $\bar{W}$ or the sets in $\bar{W}^{\prime}$. To our knowledge, this is also a property which has not yet been observed.

This concludes our remarks on examples and applications of $\mathcal{S}$ and $\mathscr{D}(\mathcal{S})$.

Conclusion. In this paper, we have considered the problem of characterizing the set $\mathscr{D}(\mathcal{S})$ induced by considering the supports of assignments in a positive cone $\mathcal{\delta}$. Utilizing the algebraic work on $\mathcal{S}$ in $\S 1$, we were able to show that $\mathscr{D}(\delta)$ was a lattice. In fact, we were able to show that $\mathscr{D}(\delta)$ was a special type of lattice which we called an ideal complemented lattice.

In $\S 2$, we considered vector space transformations on $\delta$ which were used to 
transverse this $\mathcal{S}$ in an algebraic manner. Further, the functions used for these transformations, called chains, were shown to be useful in algebraically characterizing atoms, as well as useful in obtaining other patterns in this lattice.

In $\S 3$, some local structure of $\mathscr{D}(\mathcal{S})$ was obtained by using the notions of minimal patterns and clusters. By using these notions, we were able to show that $\mathscr{D}(\mathcal{S})$ was covered by Boolean lattices of intervals $[K, \operatorname{cl}(K)]$ where $K$ was minimal.

In the final section, the goal was simply to verify that the special lattice introduced in this paper was not contrived but, in fact, was the lattice of many often studied combinatorial structures. For this, we offered irreducible and fully indecomposable matrices, strongly connected digraphs, and positive solutions to linear systems of equations as studied in linear programming.

The work in this paper then does not completely characterize the lattice $\mathscr{D}(\delta)$, however, it does introduce the lattice and supply some of its basic characteristics. The global characterization of $\mathscr{D}(\mathcal{S})$, however, seems rather complex and will no doubt require some further inventive tools, such as minimal patterns and clusters, for its total description.

\section{REFERENCES}

1. D. J. Hartfiel and J. W. Spellmann, A role for doubly stochastic matrices in graph theory, Proc. Amer. Math. Soc. 36 (1972), 389-394. MR 47 \# 4844.

2. Richard Sinkhorn and Paul Knopp, Problems involving diagonal products in nonnegative matrices, Trans. Amer. Math. Soc. 136 (1969), 67-75. MR 38 \#2151.

Department of Mathematics, Texas A\&M University, College Station, Texas 77843 